\title{
Case report: two human Streptococcus suis infections in Borneo, Sabah, Malaysia
}

\author{
Giri Shan Rajahram ${ }^{1,2^{*}}$, Ahneez Abdul Hameed ${ }^{3}$, Jayaram Menon ${ }^{2,4}$, Timothy William5, \\ Paul Anantharajah Tambyah ${ }^{6}$ and Tsin Wen Yeo ${ }^{7,8,9}$
}

\begin{abstract}
Background: Streptococcus Suis (S.suis) is increasingly being recognised as a potentially preventable emerging zoonotic infection in humans with a global distribution. It is a major cause of meningitis especially among those in contact with pigs and has also been associated with a toxic shock syndrome.

Case presentations: We report the first two human cases from Sabah, Borneo, Malaysia which expands the global reach of this important pathogen. Here, we illustrate their epidemiological risk factors, clinical presentation and resulting sequelae of both patients.

Conclusion: The continued public health threat of zoonotic infections such as S.suis, highlights the need for accurate epidemiological surveillance, regulation of pig farming, slaughtering and continued advocacy of best practices for pork preparation and consumption.
\end{abstract}

Keywords: Streptococcus suis, Meningitis, Sabah, Case report

\section{Background}

Streptococcus suis (S.suis) is a porcine zoonotic infection which is increasingly recognised. Meningitis and septicaemia have been described as the most common clinical syndromes [1]. Since the first reported human case in Denmark, increasing numbers have been reported worldwide [2]. In South East Asia, Thailand and Vietnam have reported the largest number of cases with sporadic reports from Singapore, Philippines and Laos [2]. To date, there have not been any cases reported from Malaysia or Indonesia partly because of the limited pig rearing due to cultural reasons. We report two cases from Sabah, Borneo, Malaysia.

\section{Case report}

The first patient was a 41-year-old previously healthy man who presented with 2 day history of fever, headache, vomiting, neck pain and behavioural change. He reared four pigs at home and had daily contact with them. On

\footnotetext{
* Correspondence: gsrajahram@gmail.com

${ }^{1}$ Infectious Diseases Unit, Department of Medicine, Queen Elizabeth Hospital,

Kota Kinabalu, Sabah, Malaysia

${ }^{2}$ Clinical Research Centre, Queen Elizabeth Hospital, Kota Kinabalu, Sabah,

Malaysia

Full list of author information is available at the end of the article
}

examination, he was confused and disorientated, with a Glasgow Coma Scale (GCS) 7/15, hypotensive with a blood pressure of $89 / 57 \mathrm{mmHg}$, and had meningeal signs, however there was no rash noted. He was intubated for airway protection, started on noradrenaline, ceftriaxone and acyclovir. Blood tests indicated leucocytosis $(13.7 \times$ $10^{9}$ white blood cell/L), neutrophilia $\left(12.63 \times 10^{9}\right.$ neutrophils/L), lymphopenia $\left(0.72 \times 10^{9}\right.$ lymphocytes/L), elevated C-reactive protein $(320 \mathrm{mg} / \mathrm{L})$, and hypoalbuminemia (28 g/L). Contrast enhanced computed tomography of the brain and transthoracic echocardiogram were normal. Lumbar puncture done $12 \mathrm{~h}$ after admission and initial antibiotic administration showed an opening pressure of $27 \mathrm{cmH}_{2} \mathrm{O}$ and the cerebrospinal fluid (CSF) was noted to be cloudy with a protein level of $2.16 \mathrm{~g} / \mathrm{L}$, a glucose level of $1.98 \mathrm{mmol} / \mathrm{L}$ (concomitant serum glucose was $7.6 \mathrm{mmol} / \mathrm{L}$ ), and 95 leukocytes/ $\mu \mathrm{L}$ (30\% neutrophils and $65 \%$ lymphocytes). CSF gram stain and culture did not reveal any organism however blood cultures after $3 \mathrm{~h}$ of incubation were positive for gram positive cocci in chains. Culture specimens were plated on blood agar and after $18 \mathrm{~h}$ of incubation in $37^{\circ} \mathrm{C}$ under aerobic conditions, the growth showed alpha haemolytic colonies which were catalase negative. These colonies were identified using matrix-assisted laser desorption ionization time-of-flight 
mass spectrometry (MALDI-TOF MS) VITEK $^{\circ}$ MS MALDI-TOF MS (bioMériux) as Streptococcus suis with a 99.9\% probability score. Antibiotic susceptibility testing was carried out according to the Clinical Science Laboratory Institute [3] testing recommendation using the Kirby-Bauer method. The strain was sensitive to penicillin and ceftriaxone the minimal inhibitory concentration (MIC) of penicillin and ceftriaxone were identified by epsilometer test (E-test bioMériux) with an MIC $<0.12 \mathrm{ug} / \mathrm{ml}$ and $<1.0 \mathrm{ug} / \mathrm{ml}$ respectively). The patient was extubated after $48 \mathrm{~h}$ and continued to make a good clinical recovery with antibiotics and completed a two week course of ceftriaxone. However, in the second week of admission he developed tinnitus with reduced hearing in both ear associated with vertigo, loss of balance and nausea. He was scheduled for a pure tone audiometry on clinic follow up upon discharge however defaulted his appointment and we do not have any record of permanent eighth nerve damage.

The second patient was a 44-year-old man, presented with a 2 day history of fever with chills and rigors associated with headache and vomiting. On examination he was alert and conscious with no signs of meningism. He had no fever on admission, the blood pressure was 124/ $68 \mathrm{mmHg}$ and heart rate 81 beats per minute. Systemic examination was unremarkable. He worked as a butcher in local market handling pork and had injured his thumb while slaughtering a pig two days prior to onset of symptoms. Blood tests showed leucocytosis $\left(35.4 \times 10^{9}\right.$ white blood cell/L), neutrophilia $\left(33.04 \times 10^{9}\right.$ neutrophils/L), lymphopenia $\left(0.70 \times 10^{9}\right.$ lymphocytes/L), elevated C-reactive protein $(226.8 \mathrm{mg} / \mathrm{L})$, and hypoalbuminemia $(29 \mathrm{~g} / \mathrm{L})$. Blood culture on admission and antibiotic susceptibility testing performed by methods described above was positive for Streptococcus suis which was sensitive to penicillin. Lumbar puncture performed $48 \mathrm{~h}$ after admission was normal with no white blood cells and normal glucose and protein. His echocardiogram also did not show any evidence of endocarditis. He completed two weeks of intravenous penicillin $G$ and made complete recovery on discharge without any residual neurological or otological deficits.

\section{Discussion}

Consistent with previous reports $[1,2,4]$ both our patients had significant exposure to pigs prior to developing infection. In Asia, besides direct trauma as with our second patient, it is increasing recognised that suboptimal preparation and consumption of pork and its organs may increase the risk of acquiring this disease $[1,2,4]$.

The first patient presented with meningitis associated with bacteraemia and developed hearing loss and vestibular symptoms during the course of admission. This is consistent with previous reports of $S$. suis meningitis with labyrinthitis [5] and sensory neural hearing loss occurring in up to $2 / 3 \mathrm{rd}$ of patients with a guarded prognosis for recovery [4]. The use of dexamethasone therapy in association to reducing hearing loss is controversial, with one study showing reduction in severe hearing loss [4] and a subsequent case series demonstrating hearing loss continued to occur despite therapy [6]. As with other forms of bacterial meningitis, the best outcomes are likely to be obtained by early recognition and rapid administration of parenteral antibiotics, combined administration of dexamethasone patients with a history of porcine exposure, and in regions with a high prevalence of S.suis meningitis [7]. Referral to the Otorhinolaryngologist for audiometry and vestibular testing may be needed including early evaluation for cochlear implant [8].

Although meningitis is the most common described presentation of S.suis infection, sepsis, uveitis, endoptahlmitis, endocarditis, arthritis and spondylodiscitis have all been previously reported $[1,2]$. Of note, large outbreaks in China have described streptococcal toxic shock syndrome (STSS) associated with S. suis infection with rapid clinical deterioration including purpura fulminans and high mortality $[9,10]$. While this syndrome has not been recognised outside of China, there is a concern that limited diagnostic resources and a lack of accessibility to healthcare may be the reason why this is so in other parts of Asia.

To date, 29 serotypes S.suis have been described [11]. The majority of human cases have been attributed to serotype 2 with also some sporadic cases from other serotypes, mainly serotype $14^{10}$. Reliable multiplex PCR methods are replacing traditional biochemical based methods in detecting these serotype [12], this coupled with multilocus sequence typing (MLST) this has led to better epidemiological characterization and improved understanding virulence patterns of this organism [11]. Unfortunately, these were not available in our regional hospital as the need for specialised expertise and funding limits such testing to regional and national reference laboratories or in an academic setting.

\section{Conclusion}

In summary our cases illustrate the common clinical presentation and sequelae associated with Streptococcus suis infection. Although it is a common zoonotic infection in certain parts of Asia, the true burden of illness in Malaysia in not know. The continued public health threat of zoonotic infections such as S.suis, highlights the need for accurate epidemiological surveillance, regulation of pig farming, slaughtering and continued advocacy of best practices for pork preparation and consumption.

\section{Abbreviations}

CSF: Cerebrospinal fluid; MALDI-TOF MS: Matrix-assisted laser desorption ionization time-of-flight mass spectrometry; MIC: Minimal inhibitory concentration; MLST: Multilocus sequence typing; S.suis: Streptococcus suis; STSS: Streptococcal toxic shock syndrome 


\section{Acknowledgments}

The authors would like to thank the Director General of Health, Malaysia for permission to publish this report.

We would also like to thank Dr. Goh Pik Pin and Clinical Research Centre, Malaysia for their continued support to publish this report.

\section{Funding}

Nil.

\section{Availability of data and materials}

All data are available in the patients' admission records to the hospital.

\section{Authors' contributions}

GSR, AAH, YTW, TW, and PAT prepared the manuscript. All authors read and approved the final manuscript.

\section{Competing interests}

The author(s) declare that they have no competing interests.

\section{Consent for publication}

Verbal informed consent was obtained from the patients for publication of this report.

\section{Ethics approval and consent to participate}

Not applicable.

\section{Author details}

'Infectious Diseases Unit, Department of Medicine, Queen Elizabeth Hospital, Kota Kinabalu, Sabah, Malaysia. ${ }^{2}$ Clinical Research Centre, Queen Elizabeth Hospital, Kota Kinabalu, Sabah, Malaysia. ${ }^{3}$ Microbiology Unit, Department of Pathology, Queen Elizabeth Hospital, Kota Kinabalu, Sabah, Malaysia. ${ }^{4}$ Department of Medicine, Queen Elizabeth Hospital, Kota Kinabalu, Sabah, Malaysia. ${ }^{5}$ Jesselton Medical Centre, Kota Kinabalu, Sabah, Malaysia. ${ }^{6}$ Department of Medicine, Yong Loo Lin School of Medicine, National University Health System, National University of Singapore, Singapore, Singapore. ${ }^{7}$ Lee Kong Chian School of Medicine, Nanyang Technological University, Singapore, Singapore. ${ }^{8}$ Institute of Infectious Disease and Epidemiology, Tan Tock Seng Hospital, Singapore, Singapore. ${ }^{9}$ Global Health Division, Menzies School of Health Research, Darwin, Australia.

Received: 16 December 2016 Accepted: 28 February 2017

Published online: 04 March 2017

\section{References}

1. Huong VTL, Ha N, Huy NT, Horby P, Nghia HDT, Thiem VD, et al. Epidemiology, clinical manifestations, and outcomes of Streptococcus suis infection in humans. Emerg Infect Dis. 2014;20:1105-14.

2. Hughes JM, Wilson ME, Wertheim HF, Nghia HD, Taylor W, Schultsz C. Streptococcus suis: an emerging human pathogen. Clin Infect Dis. 2009;48(5): 617-25. doi:10.1086/596763.

3. CLSI. Performance Standards for Antimicrobial Susceptibility Testing. 26th ed. CLSI Supplement M100S. Wayne: Clinical and Laboratory Standards Institute; 2016.

4. Mai NT, Hoa NT, Nga TV, le Linh D, Chau TT, Sinh DX, et al. Streptococcus suis meningitis in adults in Vietnam. Clin Infect Dis. 2008:46:659-67. doi:10.1086/527385. pmid:19413493

5. Donsakul K, Dejthevaporn C, Witoonpanich R. Streptococcus suis infection: clinical features and diagnostic pitfalls. Southeast Asian J Trop Med Public Health. 2003:34:154-8.

6. van Samkar A, Brouwer MC, Schultsz C, van der Ende A, van de Beek D. Streptococcus suis meningitis in the Netherlands. J Infect. 2015. doi: 10.1016/ j.jinf.2015.07.001. pmid:26165610

7. van Samkar A, Brouwer MC, Schultsz C, van der Ende A, van de Beek D. Streptococcus suis meningitis: a systematic review and meta-analysis. PLoS Negl Trop Dis. 2015;9(10):e0004191. doi:10.1371/journal.pntd.0004191.

8. Barbosa MH, Felix F, Ribeiro MG, Tomita S, Pinheiro C, Baptista MM. Profile of patients assessed for cochlear implants. Braz J Otorhinolaryngol. 2014:80:305-10. doi:10.1016/j.bjorl.2014.05.011. pmid:25183180.

9. $\mathrm{Yu} \mathrm{H}$, Jing $H$, Chen Z, Zheng H, Zhu X, Wang H, et al. Human Streptococcus suis infection outbreak, Sichuan, China. Emerg Infect Dis [2006 Jun [date cited]. http://dx.doi.org/10.3201/eid1206.051194
10. Tang J, Wang C, Feng Y, Yang W, Song H, Chen Z, et al. Correction: Streptococcal Toxic Shock Syndrome Caused by Streptococcus suis Serotype 2. PLoS Med. 2006;3(8):e377. doi:10.1371/journal.pmed.0030377.

11. Goyette-Desjardins G, Auger JP, Xu J, Segura M, Gottschalk M. Streptococcus suis, an important pig pathogen and emerging zoonotic agent-an update on the worldwide distribution based on serotyping and sequence typing. Emerg Microbes Infect. 2014;3:e45.

12. Kerdsin A, Akeda Y, Hatrongjit R, Detchawna U, Sekizaki T, Hamada S. Streptococcus suis serotyping by a new multiplex PCR. J Med Microbiol. 2014;63:824-30

\section{Submit your next manuscript to BioMed Central and we will help you at every step:}

- We accept pre-submission inquiries

- Our selector tool helps you to find the most relevant journal

- We provide round the clock customer support

- Convenient online submission

- Thorough peer review

- Inclusion in PubMed and all major indexing services

- Maximum visibility for your research

Submit your manuscript at www.biomedcentral.com/submit
Biomed Central 